\title{
Topical Sevoflurane: An Alternative Treatment for Pressure Ulcers
}

\author{
Isin Gencay \\ Department of Anaesthesiology and Reanimation, Faculty of Medicine, Kirikkale University, Yahsihan Kirikkale, Turkey
}

\begin{abstract}
Pressure ulcers are a common problem in intensive care unit (ICU). Recently, topical sevoflurane has been used especially for treatment of venous ulcers and infected skin ulcers. We present a case of topical sevoflurane treatment of a pressure ulcer. Treatment was applied for one month without any antibiotic supplementation. After one month, the ulcer size was decreased, necrotic tissues were cleared, and wound cultures were negative. There are three beneficial effects of local sevoflurane treatment: good analgesic action, antibiotic effects, and acceleration of wound healing. These beneficial effects may make sevoflurane an alternative treatment for pressure ulcers in an ICU.
\end{abstract}

Key Words: Ulcer, Wound care, Topical antioxidants.

How to cite this article: Isin G. Topical sevoflurane: An alternative treatment for pressure ulcers. J Coll Physicians Surg Pak $2019 ; 29$ (Supplement 2):S92-S94.

\section{INTRODUCTION}

The pressure ulcer is a wound resulting from ischemia of tissue due to continuous pressure on protruding parts of the body. It has become a common health problem in long-term hospitalised and immobilised patients. Intensive care unit (ICU) patients are at greater risk for pressure ulcers than other patients because they have multiple comorbidities, and many of them are treated with anesthetic agents in order to maintain sedation and analgesic effects. These factors impair the ability of the patient to identify the location of the ischemic discomfort. The risk of developing pressure ulcers should be identified when patients are admitted to the ICU. There have been numerous scales to determine these risks. These consist of nutritional status, skin moisture, friction, mobility, and sensory perception. Pressure ulcers may cause many problems such as infections, pain, discomfort, and prolonged hospital stay. The main consideration should be the prevention of pressure ulcers. ${ }^{1}$ Many protocols and strategies have been developed in order to prevent pressure ulcers. However, these strategies are not enough to avoid pressure ulcers, and treatment protocols become essential. Treatment protocols consist of local wound care, debridement, moisture balance, control of inflammation, and infection. There are many advanced treatments, and new protocols have also been used. ${ }^{2}$ Sevoflurane is a halogenated derivative of diethyl ether and a commonly used liquid volatile anesthetic agent for induction or maintenance of anesthesia. ${ }^{3}$ Recently, sevoflurane has

Correspondence to: Dr. Isin Gencay, Department of

Anaesthesiology and Reanimation, Faculty of Medicine,

Kirikkale University, Yahsihan Kirikkale, Turkey

E-mail:snyzc@yahoo.com

Received: April 12, 2019; Revised: June 27, 2019;

Accepted: July 10, 2019 become widely used for venous ulcer treatments. ${ }^{4}$ In this report, we aim to present the treatment of a pressure ulcer with topical sevoflurane.

\section{CASE REPORT}

A 76-year female was admitted to ICU after cardiopulmonary arrest with a primary diagnosis of chronic obstructive respiratory disease. The patient was followed with a Glasgow Coma Score (GCS) of 4. Mechanical ventilation support was maintained for two months. After one month, a pressure ulcer had developed at the upper sacral region. Two months later, the ulcer was grade 3 , measured $5 \times 7 \mathrm{~cm}\left(35 \mathrm{~cm}^{2}\right)$, and necrotic fields were observed (Figure 1). At this time, wound culture revealed pseudomonas aeruginosa that was susceptible to colistin. The C-reactive protein (CRP) was $65 \mathrm{mg} / \mathrm{L}$ and the white blood cell (WBC) count was $18,000 / \mathrm{mm}^{3}$. However, there were no infectious symptoms like redness, swelling or purulent appearance. After written consent from the patient's relatives, topical sevoflurane irrigation was initiated every two days for a period of one month at a dose of $1 \mathrm{ml} / \mathrm{cm}^{2}$.

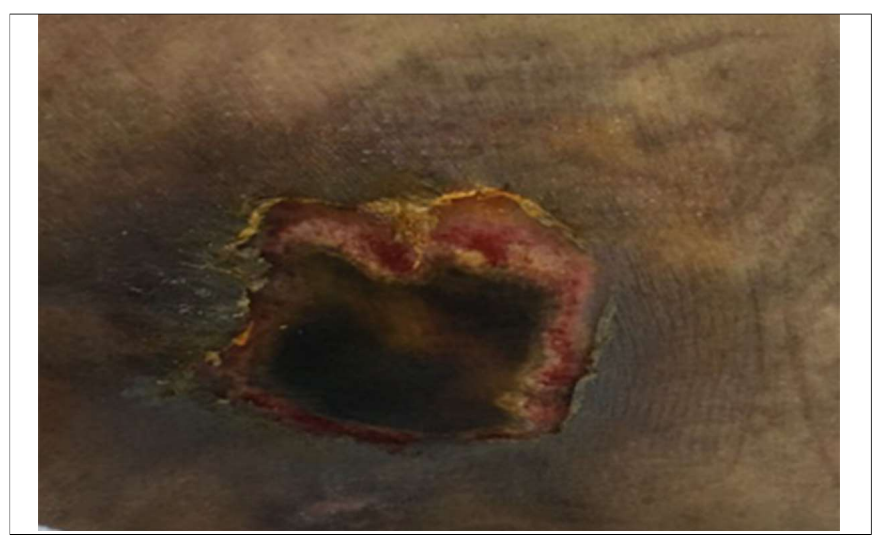

Figure 1: Sacral grade 3 pressure ulcer, before treatment. 


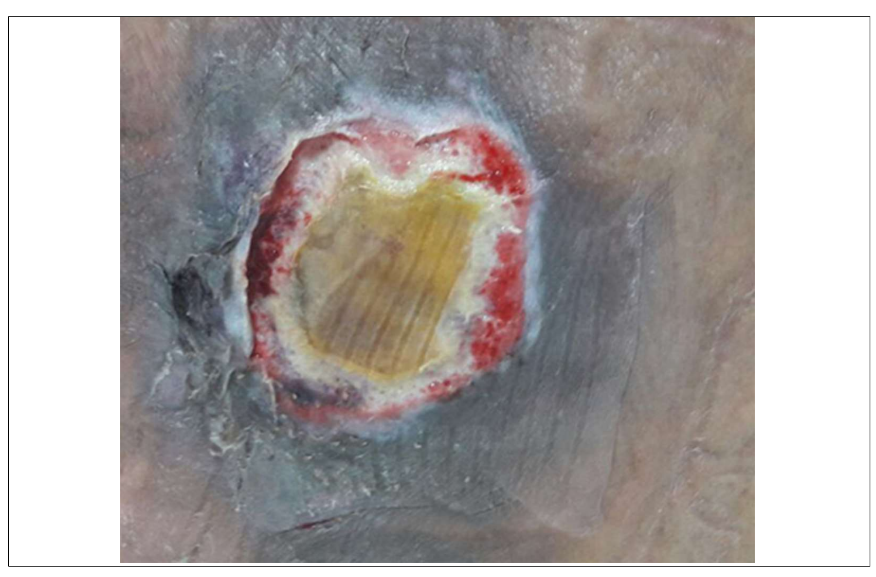

Figure 2: Sacral pressure ulcer after treatment.

Irrigation was done carefully at the wound site, and contact of the sevoflurane with the healthy skin tissue was avoided. After 30 days, another sample was taken from the wound for culture evaluation and it was negative. At that time, CRP was $50 \mathrm{mg} / \mathrm{L}$ and WBC count was $15,000 / \mathrm{mm}^{3}$. The wound size measured $4 \times 5 \mathrm{~cm}$ $\left(20 \mathrm{~cm}^{2}\right)$, and epithelialisation with granulation tissue could be clearly observed at the edges of the wound. Moreover, a decrease in necrotic fields was clearly observed (Figure 2). Further treatment of the pressure ulcer with sevoflurane was planned.

\section{DISCUSSION}

Topical sevoflurane administration has been studied previously for chronic venous ulcer treatment and for infected surgical wounds. The analgesic effects of sevoflurane in leg ulcers, surgical wounds, malignant leg ulcers, and epidural abscesses with cutaneous fistulation have been reported. ${ }^{3-5}$ Moya et al. found that sevoflurane had long-lasting analgesic effects in patients with painful chronic venous ulcers. ${ }^{6}$ Gines et al. demonstrated that topical sevoflurane decreased opioid consumption in patients with chronic vascular skin ulcers. ${ }^{7}$ The mechanism of its analgesic effects is not fully understood. It has been suggested that topical sevoflurane has a peripheral analgesic effect by partial pressure on the peripheral nociceptors that block pain transmission. 6 The use of topical sevoflurane for wound care provides analgesic effects that ensure comfort for patients suffering from pressure ulcers. However, we were not able to observe the analgesic effects of topical sevoflurane, because our patient received sedative and analgesic treatment in the intensive care unit.

The antibiotic effects of sevoflurane have been demonstrated especially on pathogens resistant to conventional antibiotics. The antibacterial effects of sevoflurane on S.aureus, P.aeruginosa, and E.coli have been reported previously. Serrano and colleagues showed the antibacterial effects of both isoflurane and sevoflurane on pathogens resistant to conventional antibiotics. ${ }^{8}$
Martinez et al. reported a case of a 43-year patient with a surgical site wound superinfected with P.aeruginosa and S.aureus, treated with topical sevoflurane. ${ }^{5}$ Moya et al. treated an infected chronic venous ulcer with topical sevoflurane for one month without systemic antibiotic treatment, and Methicillin-resistant staphylococcus aureus (MRSA) infection showed progressive improvement. 4 These studies propose that sevoflurane primarily affects the cell wall of the bacteria and it also may have a synergistic effect with conventional antibiotics.4,5 However, Rahman et al. found that clinical relevant doses of sevoflurane had no effect on growth of P.aeruginosa and S.aureus. ${ }^{9}$ Karabiyik et al. assessed the antibacterial effects of sevoflurane and nitrous oxide and concluded that the antibacterial effects of inhalation anaesthetics depend on the duration of anesthesia and type of micro-organism. ${ }^{10}$ In our case, the infection symptoms such as, local signs and systemic fever were missing and only laboratory findings showed positive wound culture. Nevertheless, P.aeruginosa growth in wound culture turned into negative after topical sevoflurane treatment without any systemic antibiotic treatment. However, this outcome is not sufficient to validate the antibiotic effects of topical sevoflurane.

Topical sevoflurane administration has been shown to improve healing in venous ulcers. Sevoflurane action on wound healing is unknown, but it has been suggested that its vasodilatory effects increase the microcirculation. It may have a directly inhibitory effect on vascular smooth muscle that results in vasodilatation. ${ }^{6}$ However, Lee and colleagues suggested that sevoflurane exposure can alter the inflammatory phase of the healing process. ${ }^{11}$ Gines et al. found a $51.1 \%$ reduction in ulcer size with 90 days of treatment of topical sevoflurane. ${ }^{7}$ We observed an approximately $42 \%$ decrease in ulcer size with one month of treatment with topical sevoflurane.

Topical sevoflurane can cause irritant effects on healthy skin, so avoiding contact to the healthy skin is important. Otherwise, it is safe and well tolerated. ${ }^{12}$ The use of topical sevoflurane in pressure ulcer treatment is not very common. It can be a good alternative for traditional pressure ulcer treatment and should be recognised.

Pressure ulcers continue to be a persistent and complicated problem in the ICU. Topical sevoflurane can be a good and a safe therapeutic alternative, for pressure ulcers.

\section{PATIENT'S CONSENT:}

Informed consent was obtained by the patient's first degree relatives.

\section{CONFLICT OF INTEREST:}

Author declared no conflict of interest.

\section{AUTHOR'S CONTRIBUTION:}

IG: Planned, applied and written this case report. 


\section{REFERENCES}

1. Smit I, Harrison L, Letzkus L, Quatrara B. What factors are associated with the development of pressure ulcers in a medical intensive care unit? Dimens Crit Care Nurs 2016; 35:37-41.

2. Sibbald RG, Goodman L, Norton L, Krasner DL, Ayello EA. Prevention and treatment of pressure ulcers. Skin Therapy Lett 2012; 17:4-7.

3. Ferrara P, Domingo-Chiva E, Selva-Sevilla C, Campos-García J, Gerónimo-Pardo M. Irrigation with liquid sevoflurane and healing of a postoperative, recurrent epidural infection: A potential cost-saving alternative. World Neurosurg 2016; 90: 702.e1-702.e5.

4. Imbernón-Moya A, Ortiz-de Frutos FJ, Sanjuan-Alvarez M, Portero-Sanchez I, Merinero-Palomares R. Topical sevoflurane for chronic venous ulcers infected by multi-drug-resistant organisms. Int Wound J 2017; 14:1388-90.

5. Rueda-Martínez JL, Gerónimo-Pardo M, Martínez-Monsalve A, Martínez-Serrano M. Topical sevoflurane and healing of a postoperative surgical site superinfected by multi-drug-resistant pseudomonas aeruginosa and susceptible staphylococcus aureus in an immunocompromised patient. Surg Infect (Larchmt) 2014; 15:843-6.

6. Imbernon-Moya A, Ortiz-de Frutos FJ, Sanjuan-Alvarez M, Portero-Sanchez I, Merinero-Palomares R. Pain, quality of life, and functional capacity with topical sevoflurane application for chronic venous ucers: A retrospective clinical study. EJVES Short Rep 2017; 36:9-12.

7. Dámaso Fernández-Ginés $F$, Cortiñas-Sáenz $M$, MateoCarrasco $\mathrm{H}$, de Aranda AN, Navarro-Muñoz E. Efficacy and safety of topical sevoflurane in the treatment of chronic skin ulcers. Am J Health Syst Pharm 2017; 74:e176-e182.

8. Martínez-Serrano M, Gerónimo-Pardo M, Martínez-Monsalve A, Crespo-Sánchez MD. Antibacterial effect of sevoflurane and isoflurane. Rev Esp Quimioter 2017; 30:84-89.

9. A-Rahman A, Pedler S, Bray R, O'Brien CJ. To the editor: Effect of anesthetic gases on the growth of pseudomonas aeruginosa, haemophilus influenzae, and staphylococcus aureus. Pediatr Pulmonol 2002; 34:226-7.

10. Karabiyik L, Türkan H, Ozisik T, Saraçli MA, Haznedaroglu T. Effects of sevoflurane and/or nitrous oxide on bacterial growth in in vitro culture conditions. J Anesth 2007; 21:436-8.

11. Lee HJ, Kwon JY, Shin SW, Baek SH, Choi KU. Effect of sevoflurane on collagen production and growth factor expression in rats with an excision wound. Acta Anaesthesiol Scand 2010; 54:885-93.

12. Imbernón A, Blázquez C, Puebla A, Churruca M, Lobato A. Chronic venous ulcer treatment with topical sevoflurane. Int Wound J 2016; 13:1060-2. 